\title{
Joint Diagnostic of the Surface Air Temperature in Southern South America and the Madden-Julian Oscillation
}

\author{
Gustavo NAUmAnN AND WALTER M. VARGaS \\ National Scientific and Technological Research Council (CONICET), and Department of Atmospheric and \\ Oceanic Sciences, Faculty of Sciences, University of Buenos Aires, Buenos Aires, Argentina
}

(Manuscript received 10 March 2010, in final form 8 April 2010)

\begin{abstract}
The objective of this research is to explore the relationship between maximum and minimum temperatures, daily precipitation, and the Madden-Julian oscillation (MJO). It was found that the different phases of the MJO show a consistent signal on winter temperature variability and precipitation in southeastern South America. Additionally, this paper explores the spatial-temporal variations of mutual information and joint entropy between temperature and the MJO. A defined spatial pattern was observed with an increased signal in northeastern Argentina and southern Brazil. In the local mutual information analysis, periods in which the mutual information doubled the average values were observed over the entire region. These results indicate that these connections can be used to forecast winter temperatures with a better skill score in situations where both variables covary.
\end{abstract}

\section{Introduction}

The Madden-Julian oscillation (MJO) is the dominant phenomenon in the intraseasonal variability of the tropical atmosphere. This oscillation is responsible for the variability in these regions and its influence extends over important atmospheric and oceanic parameters. The typical MJO cycle is approximately 30-60 days long (Madden and Julian 1971, 1972, 1994; Zhang 2005). The influence of atmospheric circulation anomalies extends from the region under the direct influence of this phenomenon, affecting global circulation patterns. Diverse studies have been developed that relate the interaction between variations within the synoptic scale and the MJO.

Matthews and Kiladis (1999) studied the interaction between high-frequency transient disturbances and convection with the MJO. They found evidence that the propagation of high-frequency waves in the Indian Ocean could be of relevance for the onset of convective anomalies on an intraseasonal scale. For tropical-extratropical interactions, Matthews and Meredith (2004) and Zhou and Miller (2005) found that the variability of the atmospheric

Corresponding author address: Gustavo Naumann, F.C.E. y N., Universidad de Buenos Aires, Intendente Güiraldes 2160, Pab II, 2nd Fl., Buenos Aires C1428EGA, Argentina.

E-mail: gnaumann@at.fcen.uba.ar southern annular pattern on an intraseasonal scale shows a relation with the atmospheric variability related to the MJO during the southern winter.

Additionally, in an analysis of the relation of the MJO with temperature variability, Vecchi and Bond (2004) found a significant statistical signal and spatial coherence between the atmospheric variability at high latitudes and the convective intertropical variability at the intraseasonal scale during the Northern Hemisphere winter. Because of this coherence, a connection was inferred between the MJO and surface temperature variations at high latitudes through the effects that the MJO induces on humidity and anomalies in geopotential height in the middle troposphere. These results agree with those of Higgins and Mo (1997), Mo and Higgins (1998), and Jones (2000). Additionally, Minetti and Vargas (1997) demonstrated the existence of a modulation in the intraseasonal fluctuations of temperature anomalies in the Argentinean tropics due to the different El Niño-Southern Oscillation (ENSO) phases.

This paper explores the link between temperature anomalies in southern South America and the MJO as well as its possible implications on the joint diagnosis. Section 2 presents the daily data used for the study. The spatial distribution of the relations between the MJO and maximum and minimum temperature anomalies is presented in section 3. Joint entropy and mutual information are presented in section 4 . Section 5 shows the spatiotemporal 
behavior of the joint entropy and mutual information. Finally, section 6 summarizes our main conclusions.

\section{Data}

Series of daily maximum and minimum temperatures at 53 stations provided by the National Weather Service of Argentina and the Claris Project were selected. These series ensured a large quantity of data to produce a stable estimation of the clusters over the daily temperatures (in this case, more than 20000 values). A representative geographic distribution of stations was selected to include as many climatic regions of southern South America as possible, as well as to cover a wide latitudinal selection (covering $23^{\circ}-55^{\circ} \mathrm{S}$ ).

The Real-time Multivariate MJO series 1 and 2 (RMM1 and RMM2) bivariate indexes were used to determine the possible relationship between the MJO and atmospheric circulation in southeastern South America. These indexes are based on the first two empirical orthogonal functions (EOFs) of the combined fields of the zonal wind speeds at 850 and $200 \mathrm{hPa}$, and the outgoing longwave radiation (OLR), averaged between $15^{\circ} \mathrm{N}$ and $15^{\circ} \mathrm{S}$. The projection of this daily information on the EOFs with the annual cycle and interannual variability filtered out produces a temporal series of each main component. These reproduce the variability of the intraseasonal scale. The two main associated components that make up the multivariate index are called RMM1 and RMM2. More details concerning the construction of this index can be found in Wheeler and Hendon (2004). Additionally, the index is available online (http://www.bom.gov.au/bmrc/clfor/ cfstaff/matw/maproom/RMM/).

Based on this description, the MJO is divided into eight phases, each with an average duration of approximately 6 days. As depicted in Wheeler and Hendon (2004), in phase 1, convection of a decaying MJO event is present in the central Pacific, while enhanced convection of a growing event is evident over Africa and the western Indian Ocean. Over subsequent phases convection in the Indian Ocean builds and moves to the east. In general, the trajectory of this bivariate index is represented by orbits around the origin, which evidences a systematic propagation toward the east of the MJO. A greater amplitude of these orbits means strong MJO cycles. For periods during which the MJO's signal is weak, this is reflected as random displacements near the origin (Wheeler and Hendon 2004).

\section{The relation between surface temperature and the MJO}

Average fields of maximum and minimum temperature anomalies associated with each of the eight MJO phases showing a coherent signal were constructed to evaluate the relationship between the MJO and the surface temperatures in the region. These fields considered the events in which the MJO was active. The MJO was defined as active when the values of the amplitude of the index outperformed the upper tercile.

Additionally, given that the greatest intraseasonal signal in the region is observed during the cold season (Naumann 2010), the average fields were calculated during the southern winter, defined as the months of June-August (JJA).

Figures 1 and 2 show the average fields associated with the maximum and minimum temperatures for each MJO phase. These show that the temperature signals associated with the MJO are spatially coherent. For the minimum temperature, for example, warm anomalies are observed over the entire northern region of the domain for phases 5 and 6. For phases 2 and 3, the inverse pattern of behavior is observed, with cold anomalies in nearly the entire region, except southeastern Brazil. It is worth noting that the observed anomalies, in both the warming and cooling phases, are statistically significant (estimated with a normal test). They also exceed values of $1^{\circ} \mathrm{C}$ in almost all regions, even reaching average values of $4^{\circ} \mathrm{C}$.

A coherent pattern of behavior also exists in what is observed for the maximum temperature (Fig. 1) and precipitation (Naumann 2010), principally in the northeastern portion of the region. In phases 3 and 5, negative maximum temperature anomalies (positive precipitation anomalies) were observed in the east-central portion of the region, while positive (negative) anomalies exist in Patagonia and the central Andes. Phases 1, 4, and 7 are characterized by the opposite behavior, with a deficit in precipitation in southeastern Brazil and northeastern Argentina. Excesses also stand out in phase 1 in Patagonia and the central mountain range.

\section{Joint entropy and mutual information}

When two discrete variables, $x$ and $y$, are considered for the same time $t$, it is possible to measure the degree of uncertainty or the information associated between them (Shannon 1948, 1950). The quantity that measures these properties is joint entropy $H(x, y)$. If $x$ and $y$ can assume values $m_{1}$ and $m_{2}$, respectively, then the joint entropy can be calculated as follows:

$$
H(x, y)=\sum_{x=i}^{m_{1}} \sum_{y=j}^{m_{2}} p_{i, j}(x, y) \log \left[p_{i, j}(x, y)\right],
$$

where $p_{i, j}$ represents the probability that the variable $x$ is in state $i$ while, simultaneously, the variable $y$ is in the state $j$. 


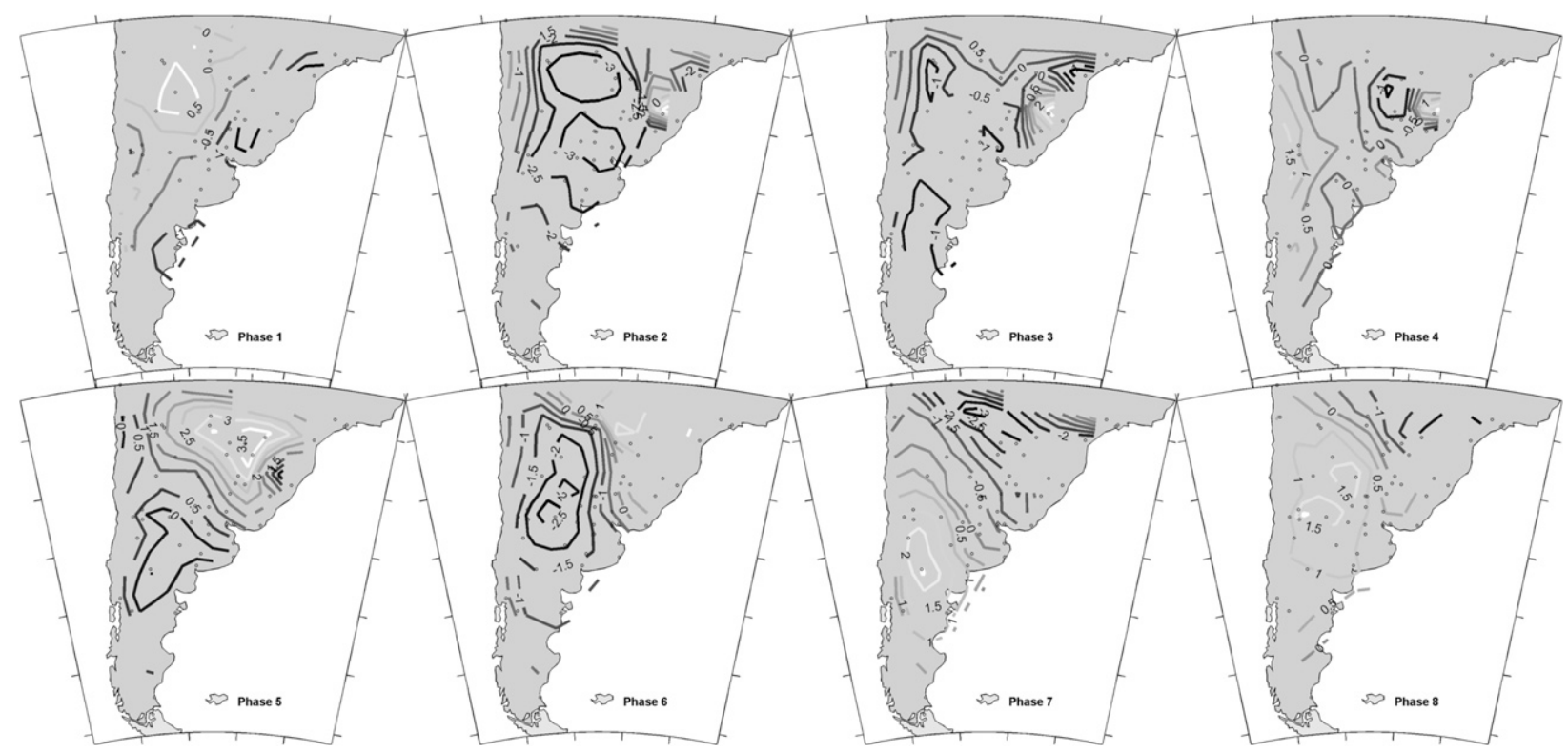

FIG. 1. Average fields of maximum winter (JJA) temperature anomalies with amplitude greater than the upper tercile for MJO phases 1-8. The dots represent the stations used in the analysis.

Joint entropy varies from minimum theoretical entropy $(H=0)$ to $\log \left(m_{1}\right)+\log \left(m_{2}\right)$. The relationship between the joint entropy and the individual entropies is

$$
H(x, y) \leq H(x)+H(y) .
$$

This relationship shows that joint entropy is always less than the sum of the entropies of each variable. The equality is only valid for the case in which variables $x$ and $y$ are independent.

Additionally, it is possible to define mutual information $(I)$ as a measure of the information shared by two variables. This quantity can be defined as a function of the individual and joint entropies of two variables, $x$ and $y$, such that

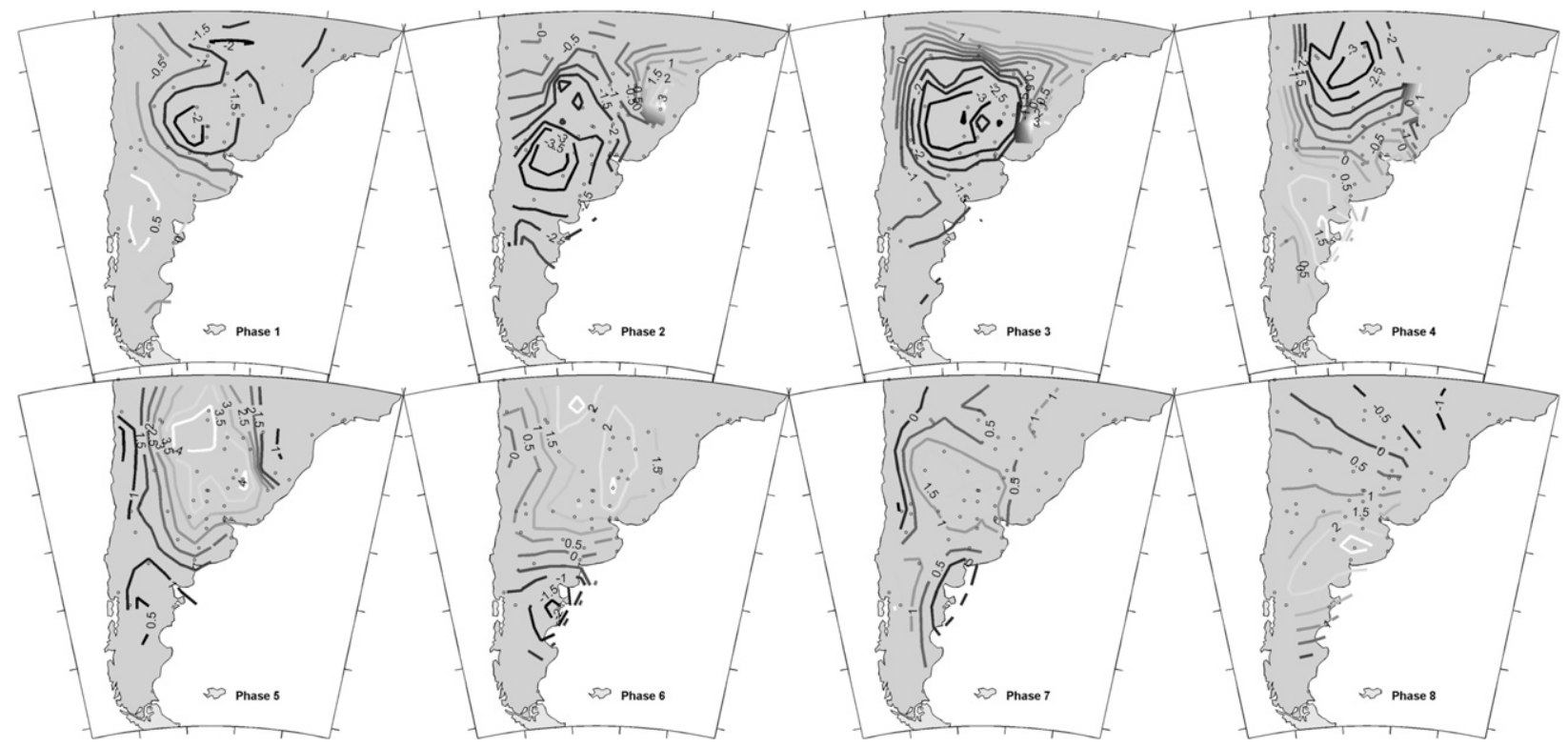

FIG. 2. As in Fig. 1, but for minimum winter (JJA) temperature anomalies. 


$$
I(x, y)=H(x)+H(y)-H(x, y) .
$$

If both variables are independent, the joint entropy is equal to the sum of the individual entropies, and, consequently, the mutual information is zero.

\section{Mutual information between temperature and the Madden-Julian oscillation}

Joint entropy and mutual information are two variables that measure the degree of shared information between variables. In the case of mutual information, this represents a measure of the reduction of entropy of a variable due to the effects of another. In other words, this represents the amount of information that two or more variables have in common. Because of this, high mutual information values indicate a large reduction of the uncertainty, while lesser $I$ values represent a small reduction in these uncertainties.

This measure of correlation between two variables can be used to examine the relationship between daily temperature and the MJO. Following this goal, the mutual information between the discrete series as a result of the bivariate classification of temperature and the phases associated with the bivariate index that represents the MJO was analyzed. Classification of the daily series of temperature produced four groups. The names of these groups were assigned according to their thermal properties (which reflect aspects of humidity, cloudiness, orography, etc.) and their association with precipitation on annual and daily scales. The groups are warm, wet, cold, and dry. The groups differentiate weather types, which represent circulation patterns. More details on bivariate temperature classification can be found in Vargas and Naumann (2008).

In this way Fig. 3 shows the spatial distribution of the mutual information in the daily temperature series discretized using cluster analysis and the MJO. Here, a southern gradient of this variable is observed with maxima in the tropical regions. Additionally, there is a maximum relationship between the two variables analyzed in southeastern Brazil and in part of the Argentinean northeast.

This result is highly related to the strengthening of convection in the equatorial Indian and Pacific Oceans. Grimm and Silva Dias (1995) found a considerable consistency among the circulation patterns (more precisely Rossby waves forced by tropical convention) observed at intraseasonal (MJO) and interannual (ENSO) time scales. Similarly, the authors found a connection in the dynamic mechanisms among 30-60-day oscillations and the southern Atlantic convergence zone (SACZ).

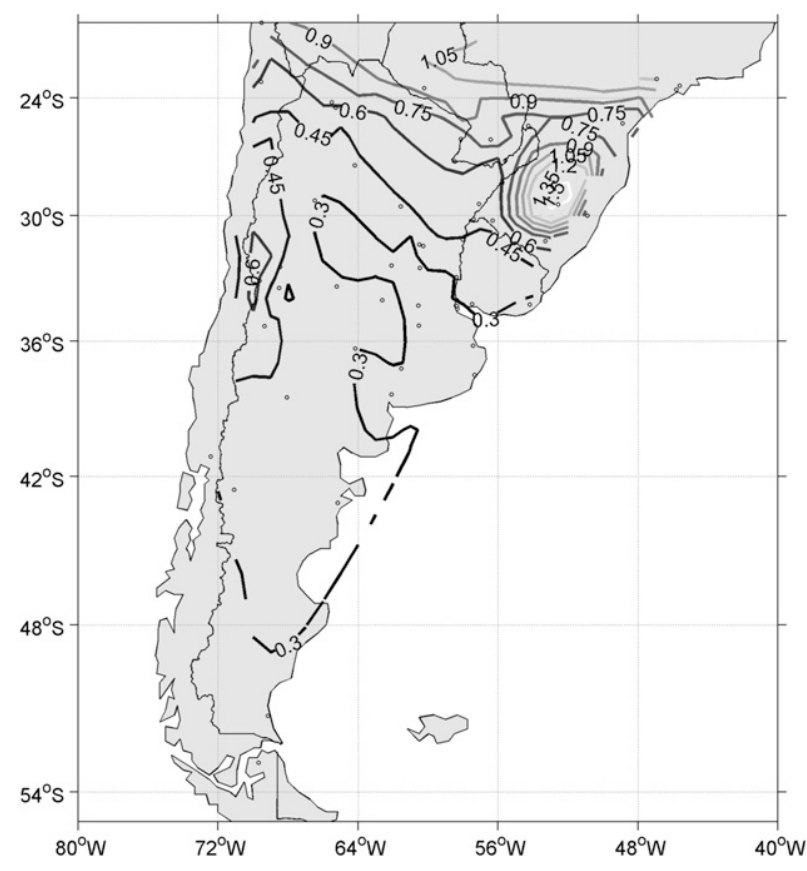

FIG. 3. Mutual information between the bivariate classification of temperature and the MJO.

These results indicate that the mutual information between temperature and the Madden-Julian oscillation is not high compared with the maximum values that can reach the variable $I$, that is, the sum of the individual entropies [Eq. (3)]. However, I reaches up to 1 bit of information in southeastern Brazil. Also, the spatial distribution of the signal is coherent with the physical processes associated with teleconnections, with the intraseasonal oscillation due to equatorial convection.

However, because the results in Fig. 3 refer to average values of mutual information and because the amplitude of the MJO varies with time, periods in which the temperature and the MJO covary to a greater or lesser extent are expected. Consequently, it is of interest to analyze the local variations of these correlations and their inferences on the overall diagnosis.

Under certain conditions, principally in the case of transitory dynamic phenomena, the analysis of average entropy fails to detect correlations existing among variables. Also, the standardized average entropy of the climate system is greater than 0.9 (Naumann and Vargas 2009). This means that, as a main property, the average predictability of the system is low (around $10 \%$ ). However, for practical applications, the average values of the different properties that define the predictability of the system are, in general, not of great usefulness. However, a specific prediction based on a finite sequence of longitude $N$ (conditional and dynamic entropy), or on the 

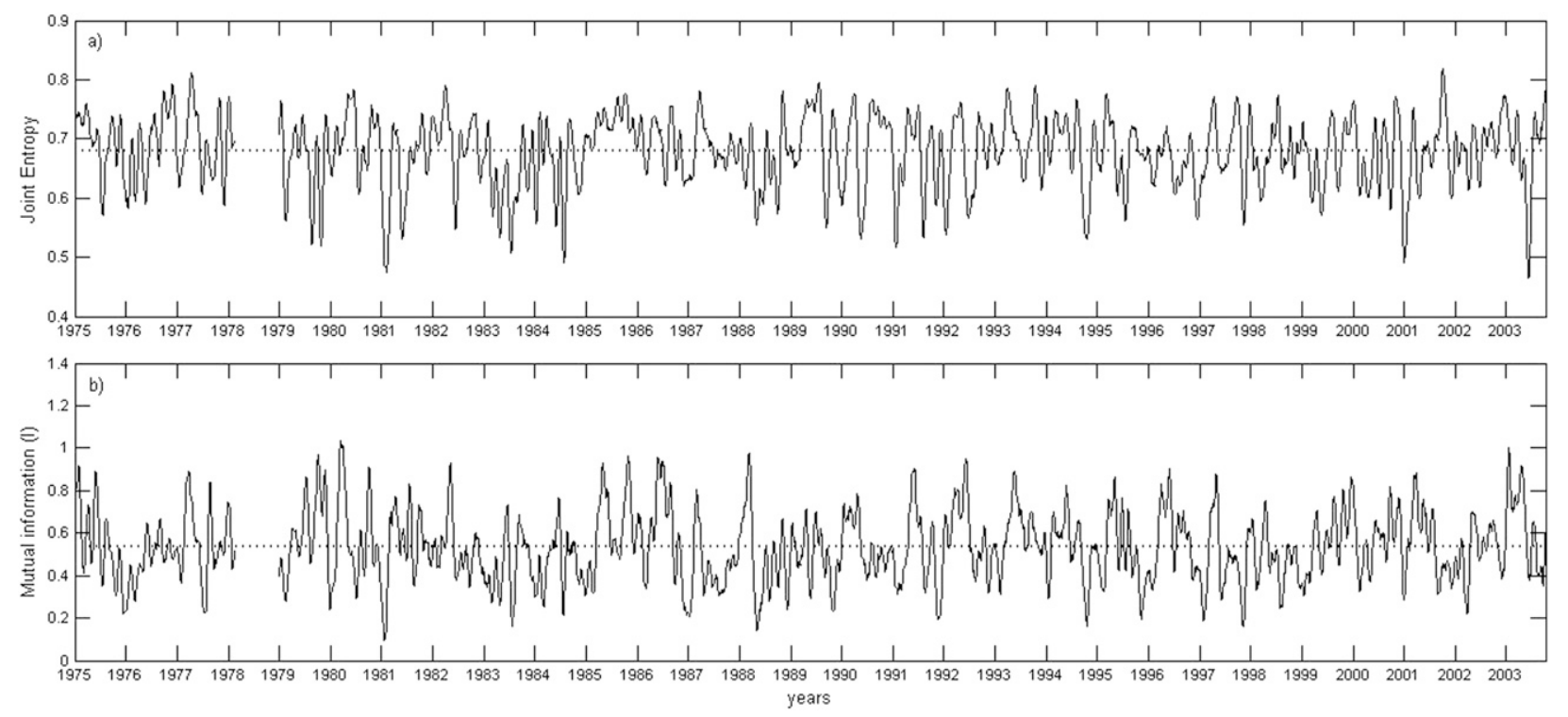

FIG. 4. (a) Standardized joint entropy and (b) mutual information between the bivariate classification of temperature in Corrientes, Argentina $\left(27.43^{\circ} \mathrm{S}, 58.74^{\circ} \mathrm{W}\right)$, and the $\mathrm{MJO}$ and its respective average (dotted line).

prediction given by the relation between two variables that share information in specific periods of time (joint entropy), could be of great importance.

Because joint entropy is a measure of dependence between two variables, it is possible to detect periods in which this measure decreases locally. This implies coherence between the processes of the two variables. Consequently, to analyze the local behavior that describes the relation between the discretized temperature and the $\mathrm{MJO}$, the joint entropy and the mutual information on 30-day mobile time windows (Figs. 4 and 5) were calculated. These show for the entire region in general that periods exist when the mutual information doubles the average values, and the standardized joint entropy decreases from values of 0.8 to less than 0.5 .

Additionally, the mutual information tends toward a defined seasonal pattern of behavior in this region. This refers to maximums of mutual information in the autumn and winter periods and minimums during the summer. These results match those found by Naumann (2010),
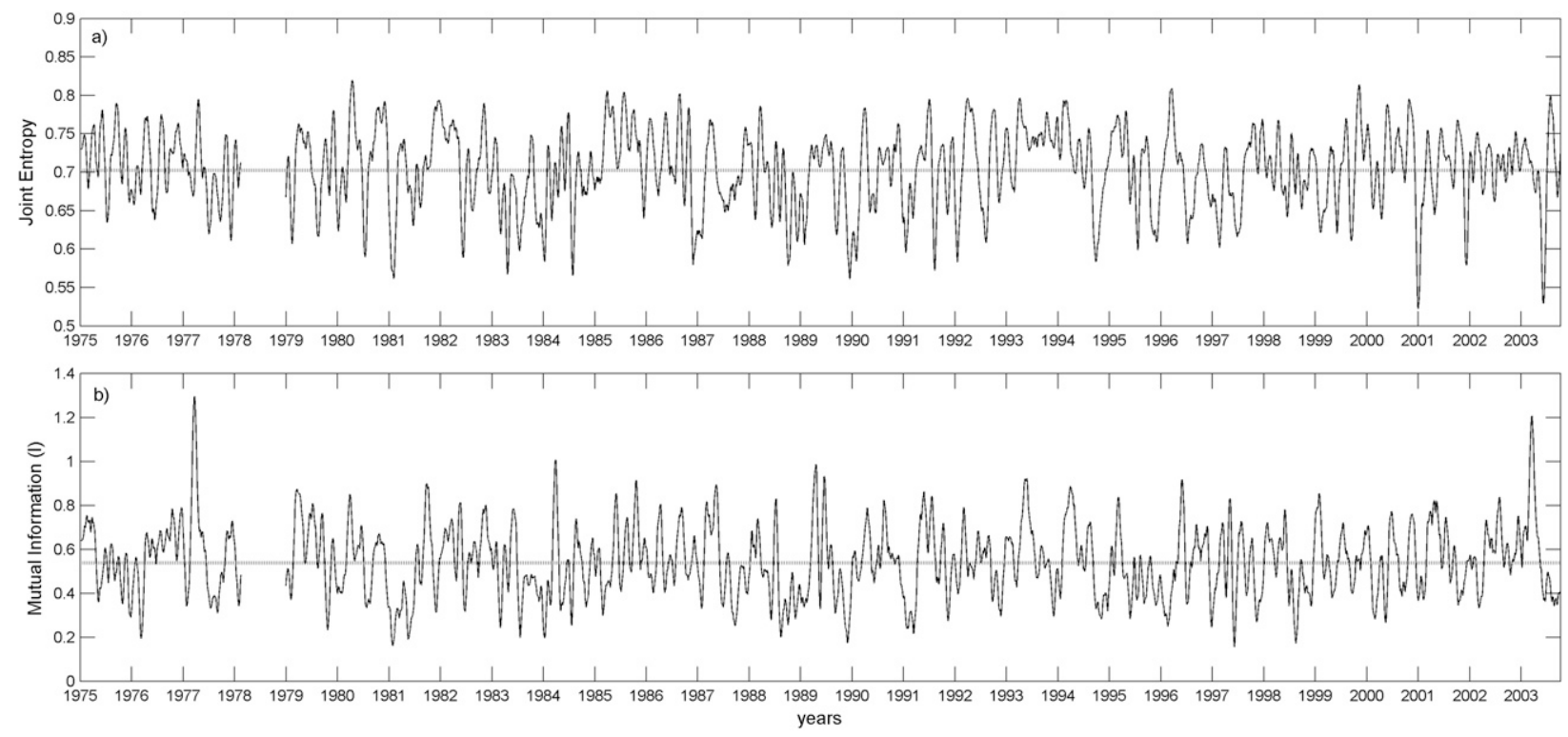

FIG. 5. As in Fig. 4, but for Pilar, Argentina $\left(31.64^{\circ} \mathrm{S}, 63.85^{\circ} \mathrm{W}\right)$. 
where the greatest intraseasonal temperature signal is observed for winter.

These results imply that for these periods, it is possible to make a diagnosis and prognosis of the temperature behavior in the region based on the analysis of the MJO variability.

\section{Conclusions}

There is a coherent signal among the distinct phases of the MJO with maximum and minimum temperatures in southeastern South America.

Because the joint entropy is a measure of the dependence between two variables, it is possible to detect periods in which this measure decreases locally, which implies a coherence between the processes of the two variables. For this reason, the joint entropy and the mutual information between the temperature and the MJO were calculated. Here, it was observed for the entire region in general that periods exist when the mutual information doubles the average values and the joint entropy decreases from values of 0.8 to less than 0.5 .

Likewise, in the localities analyzed, the mutual information tends toward a defined seasonal pattern of behavior. This refers to the maximums of mutual information in the autumn and winter seasons and the minimums during summer. These results imply that for these periods it is possible to make a diagnosis and prognosis of the temperature's behavior in the region based on the analysis of the MJO variability. Moreover, because it is now possible to predict the MJO with significant accuracy up to 15-17 days (Seo et al. 2009), it is possible to use this information to infer the temperature prognosis in the region at that time scale.

Finally, it is clear that it is necessary to investigate the local conditions in which the MJO could be a good predictor of temperatures in South America. This can be done through analysis of the joint and conditional entropy of the different joint processes that lead to extreme thermal events in the region.

Acknowledgments. This research was sponsored by Projects UBA X-228 and CONICET PIP 112-20080100762.

\section{REFERENCES}

Grimm, A. M., and P. L. Silva Dias, 1995: Analysis of tropicalextratropical interactions with influence functions of a barotropic model. J. Atmos. Sci., 52, 3538-3555.
Higgins, R. W., and K. C. Mo, 1997: Persistent North Pacific circulation anomalies and the tropical intraseasonal oscillation. J. Climate, 10, 223-244.

Jones, C., 2000: Occurrence of extreme precipitation events in California and relationships with the Madden-Julian oscillation. J. Climate, 13, 3576-3587.

Madden, R., and P. Julian, 1971: Detection of a 40-50 day oscillation in the zonal wind in the tropical Pacific. J. Atmos. Sci., 28, 702-708.

— the tropics with a 40-50 day period. J. Atmos. Sci., 29, 11091123.

— , and 1994: Observations of the 40-50-day tropical oscillation: A review. Mon. Wea. Rev., 112, 814-837.

Matthews, A. J., and G. N. Kiladis, 1999: The tropical-extratropical interaction between high-frequency transients and the MaddenJulian oscillation. Mon. Wea. Rev., 127, 661-667.

— , and M. P. Meredith, 2004: Variability of Antarctic circumpolar transport and the Southern Annular Mode associated with the Madden-Julian oscillation. Geophys. Res. Lett., 31, L24312, doi:10.1029/2004GL021666.

Minetti, J. L., and W. M. Vargas, 1997: Interaction processes between the annual wave and the disturbances in series of daily temperature. Int. J. Climatol., 10, 299-305.

Mo, K. C., and R. W. Higgins, 1998: Tropical convection and precipitation regimes in the western United States. J. Climate, 11, 2404-2423.

Naumann, G., 2010: Variabilidad intraestacional de alta y baja frecuencia de la temperatura máxima y mínima diaria en Sudamérica. Estudio de la predictabilidad mediante la entropía condicional. Ph.D. thesis, Universidad de Buenos Aires, 229 pp. [Available online at http://digital.bl.fcen.uba.ar/.]

— , and W. M. Vargas, 2009: Changes in the predictability of the daily thermal structure in southern South America using information theory. Geophys. Res. Lett., 36, L09704, doi:10.1029/ 2009GL037576.

Seo, K., W. Wang, J. Gottschalck, Q. Zhang, J. Schemm, W. R. Higgins, and A. Kumar, 2009: Evaluation of MJO forecast skill from several statistical and dynamical forecast models. J. Climate, 22 , 2372-2388.

Shannon, C. E., 1948: A mathematical theory of communication. Bell Syst. Tech. J., 27, 379-423, 623-656.

- 1950: Prediction and entropy of printed English. Bell Syst. Tech. J., 30, 50-64.

Vargas, W. M., and G. Naumann, 2008: Impacts of climatic change and low frequency variability in reference series on daily maximum and minimum temperature in southern South America. Reg. Environ. Change, 8, 45-87.

Vecchi, G. A., and N. A. Bond, 2004: The Madden-Julian oscillation (MJO) and northern high latitude wintertime surface air temperatures. Geophys. Res. Lett., 31, L04104, doi:10.1029/ 2003 GL018645.

Wheeler, M. C., and H. H. Hendon, 2004: An all-season real-time multivariate MJO index: Development of an index for monitoring and prediction. Mon. Wea. Rev., 132, 1917-1932.

Zhang, C., 2005: Madden-Julian oscillation. Rev. Geophys., 43,1-36.

Zhou, S., and A. J. Miller, 2005: The interaction of the MaddenJulian oscillation and the Arctic Oscillation. J. Climate, 18, 143-159. 